\title{
Resilient Reliable Dynamic-output-feedback Control for Takagi-Sugeno Fuzzy Time-Delayed Systems with Time-Varying Actuator Fault
}

\author{
S. Senpagam ${ }^{1}$, P. Dhanalakshmi ${ }^{2}$, R. Mohana Priya ${ }^{3}$
}

Abstract-

Keywords: T-S Fuzzy systems, Time-varying actuator failure, Fault estimation, Dynamic-output-feedback.

\section{Introduction}

In recent years, fuzzy dynamical systems in the texture of Takagi-Sugeno (T-S) model have rapid growth due to its attractive features [1]- [5]. Moreover many real-world systems exhibit nonlinear characteristics which bring severe difficulties during the process of stabilization. T-S fuzzy model based approach provides an efficient way to analyse and synthesize nonlinear systems [6][8]. On the other hand, T-S fuzzy model with time-delay cause nonlinear retarded systems. Therefore the issue of time-delay has been taken into account in T-S fuzzy model by many researchers. By constructing a novel augmented Lyapunov-Krasovskii(L-K) functional, Kwon et al. in [9] designed stability conditions for T-S fuzzy systems with time-varying delay. In [10] the problem of delay-dependent stability is discussed for T-S fuzzy systems with time-varying delay.

Obviously, many engineering systems in the real world cannot work perfectly at all times under all situations. In order to acquire higher safety and reliability criterion, the possible faults which may affect the system performance have be able to detected and identified as early as possible. To estimate such faults, observer-based fault detection, isolation and fault tolerant control using T-S fuzzy model have gained considerable interest [11]. Youssef et.al [13] designed a Proportional Integral observer for estimatng the actuator and sensor faults with unmeasurable premise variables based on T-S fuzzy model. A novel fault estimation and fault tolerant control has been proposed in [14] for T-S fuzzy sytems with time-varying state delay against actuator faults. In particular, some practical systems may have time varying faults. The fault detection technique is employed to handle these kinds of time-varying faults. With the aid of Kalman-Yakubovic-Popov lemma in a local linear model, fault detection filter system and the dynamics of filtering error generator are constructed in [15] for T-S fuzzy discrete systems. The fault detection problem for continuous-time T-S fuzzy systems in finite frequency domain against sensor faults is studied in [16]. The problem of fault detection, isolation and estimation of nonlinear systems using T-S fuzzy model is proposed in [17], wherein the minimization problem 
is formulated by bounded real lemma and Polya's theorem is used to reduce the conservatism.

In many real-world systems, state-feedback control appraoch will fail to guarantee the stabilizability when complete knowledge of the system is not known. In such cases, observer-based dynamic output feedback control is need to be designed so that the unmeasurable states can be estimated from the dynamical process. Moreover, the observer-based dynamic feedback control plays a positive role in system performance and stabilizing the unstable systems. Therefore, this control using T-S fuzzy model approach for dynamical systems has attracted remarkable attention in the control theory ( [18]- [19]). By employing the reciprocally converse approach, in [20] an investigation is made on dynamic-output-feedback control for T-S fuzzy system with time-varying input delay and output constraints. The robust dynamic output feedback control problem for a class of discrete-time nonlinear systems is investigated in [21], by employing T-S fuzzy model. External noises are common barrier for the process of stabilization of dynamical system$\mathrm{s}$, which should be controlled or removed by using some perforntance. The local $H_{\infty}$ control problem is designed in [22] for the continuous-time T-S systems where the derived LMIs are solved by means of Convex Optimization Technique. A novel robust $H_{\infty}$ dynamic sliding mode control is proposed in [24] for a class of uncertain stochastic nonlinear systems, wherein a new set of conditions guaranteeing the stochastic stability are derived in terms of LMIs. The problem of mixed $H_{\infty}$ and passivity performance analysis and design is discussed in [23] for discrete time-delay neural networks represented by T-S fuzzy model. Yet, to the authors best knowledge, the fault estimation control together with the dynamic-output-feedback and mixed $H_{\infty}$ and passivity performance has not been studied for T-S fuzzy systems. Motivated by this thought, this investigation deals on the stabilization of the T-S fuzzy time-delayed system with the incorporation of fault-tolerant dynamic-outputfeedback control via adaptive fault estimation algorithm.

\section{Problem formulation and preliminaries}

In this section our focus is to construct the closedloop system by using dynamic-output-feedback control.
Consider the following T-S fuzzy model with timevarying delay:

System rule p: If $g_{1}(x(t))$ is $\mathcal{M}_{1}^{p}$ and $g_{2}(x(t))$ is $\mathcal{M}_{2}^{p}$ and $\ldots$ and $g_{p}(x(t))$ is $\mathcal{M}_{h}^{p}$ then

$$
\begin{aligned}
\dot{x}(t)= & A_{p} x(t)+A_{d_{p}} x(t-\tau(t))+B_{p} u(t)+D_{p} \omega(t) \\
& +B_{a_{p}} f(t), \\
y(t)= & C x(t),
\end{aligned}
$$

where $p \in\{1,2, \ldots, l\}, l$ is the number of T-S fuzzy system rules, $g_{q}(x(t))$ are premises variables and $\mathcal{M}_{q}^{p}$ are fuzzy sets. Let $x(t)=\left[\begin{array}{llll}x_{1}^{T} & x_{2}^{T} & \ldots & x_{n}^{T}\end{array}\right]^{T}$ be state vector, $y(t)$ is the output signal, $\tau(t)$ denotes time-varying delay with $\tau_{1} \leq \tau(t) \leq \tau_{2}<\infty$ for some $\tau_{1} \geq 0$ and $\tau_{2}>0, u(t)$ and $\omega(t)$ stands for control input and timevarying actuator fault respectively, $f(t)$ denotes noice term and $A_{p}, A_{d_{p}}, B_{p}, B_{a_{p}} C, D_{p}$ are system parameters of appropriate dimensions. Then the over all T-S fuzzy model based on (1) can be written in the form,

$\begin{aligned} \dot{x}(t)= & \sum_{p=1}^{l} \varphi_{p}(g(t))\left\{A_{p} x(t)+A_{d_{p}} x(t-\tau(t))+B_{p} u(t)\right. \\ & \left.+D_{p} \omega(t)+B_{a_{p}} f(t)\right\}, \\ y(t)= & C x(t),\end{aligned}$

where $\varphi_{p}\left(g_{p}(x(t))\right)=\overline{\left.\left.\left.\sum_{p=1}^{l} \varpi_{p} g_{0 g_{p} x(t)}(t) t\right)\right)\right)}$, $\varpi_{p}\left(g_{p}(x(t))\right)=\Pi_{q=1}^{h} \mathcal{M}_{q}^{p}\left(g_{p}(x(t))\right), \quad g(t)=$ $\left[g_{1}^{T}(x(t)) \quad g_{2}^{T}(x(t)) \ldots g_{h}^{T}(x(t))\right]^{T}$ and $\mathcal{M}_{q}^{p}\left(g_{q}(x(t))\right)$ represents membership degree of $g_{p}(x(t))$ in $\mathcal{M}_{q}^{p}$. Let $A(\varphi)=\sum_{p=1}^{l} \varphi_{p}(g(t)) A_{p}, A_{d}(\varphi)=\sum_{p=1}^{l} \varphi_{p}(g(t)) A_{d p}$, $B(\varphi)=\sum_{p=1}^{l} \varphi_{p}(g(t)) B_{p}, B_{a}(\varphi)=\sum_{p=1}^{l} \varphi_{p}(g(t)) B_{a p}$ and $D(\varphi)=\sum_{p=1}^{l} \varphi_{p}(g(t)) D_{p}$. Then the system (2) can be rewritten in the following form:

$$
\begin{aligned}
\dot{x}(t)= & A(\varphi) x(t)+A_{d}(\varphi) x(t-\tau(t))+B(\varphi) u(t) \\
& +D(\varphi) \omega(t)+B_{a}(\varphi) f(t), \\
y(t)= & C x(t) .
\end{aligned}
$$

Taking the effects of actuator fault into account and by implementing adaptive algorithm, the fuzzy observer is constructed in the following form to estimate the faults: 
Observer rule p: If $g_{1}\left(x_{O}(t)\right)$ is $\mathcal{M}_{1}^{p}$ and $g_{2}\left(x_{O}(t)\right)$ is $\mathcal{M}_{2}^{p}$ and $\ldots$ and $g_{p}\left(x_{O}(t)\right)$ is $\mathcal{M}_{h}^{p}$ then,

$$
\begin{aligned}
\dot{x}_{O}(t)= & A_{p} x_{O}(t)+A_{d_{p}} x_{O}(t-\tau(t))+B_{p} u(t) \\
& +B_{a_{p}} f_{O}(t)+L_{p}\left[y_{O}(t)-y(t)\right], \\
y_{O}(t)= & C x_{O}(t), \\
\dot{f}_{O}(t)= & -P_{4}^{-1} F_{p}\left[\dot{e}_{y}(t)+e_{y}(t)\right],
\end{aligned}
$$

where $x_{O}(t)$ denotes observer state, $f_{O}(t)$ is estimated fault and $L_{p}$ represents observer gain to be determined. After the process of de-fuzzyfication as discussed above the observer system becomes,

$$
\begin{aligned}
\dot{x}_{O}(t) & =A(\varphi) x_{O}(t)+A_{d}(\varphi) x_{O}(t-\tau(t))+B(\varphi) u(t) \\
& +B_{a}(\varphi) f_{O}(t)+L(\varphi)\left[y_{O}(t)-y(t)\right], \\
y_{O}(t) & =C x_{O}(t), \\
\dot{f}_{O}(t) & =-P_{4}^{-1} F(\varphi)\left[\dot{e}_{y}(t)+e_{y}(t)\right],
\end{aligned}
$$

where $L_{p}$ and $F_{p}$ are the unknown gains, $P_{4}^{-1}$ represents learning rate, $L(\varphi)=\sum_{p=1}^{l} \varphi_{p}(g(t)) L_{p}, F(\varphi)=$ $\sum_{p=1}^{l} \varphi_{p}(g(t)) F_{p}$, and $e_{y}(t)=y_{O}(t)-y(t)$. Let $e(t)=$ $x_{O}(t)-x(t)$, then the error system can be expressed in the following form:

$$
\begin{aligned}
\dot{e}(t)= & {[A(\varphi)+L(\varphi) C] e(t)+A_{d}(\varphi) e(t-\tau(t)) } \\
& -D(\varphi) \omega(t)+B_{a}(\varphi) e_{f}(t) \\
e_{y}(t)= & C e(t),
\end{aligned}
$$

where $e_{f}(t)=f_{O}(t)-f(t)$.

In order to design a controller in a more effective and unbreakable way, a resilient reliable dynamic-outputfeedback fuzzy controller is designed in the following form,

Controller rule p: If $g_{1}\left(x_{c}(t)\right)$ is $\mathcal{M}_{1}^{p}$ and $g_{2}\left(x_{c}(t)\right)$ is $\mathcal{M}_{2}^{p}$ and $\ldots$ and $g_{p}\left(x_{c}(t)\right)$ is $\mathcal{M}_{h}^{p}$ then,

$$
\begin{aligned}
\dot{x_{c}}(t) & =A_{c_{p}} x_{c}(t)+B_{c_{p}} y(t), \\
u(t) & =\left(K_{p}+\delta K(t)\right) x_{c}(t)-B^{+}(\varphi) B_{a}(\varphi) f_{O}(t),
\end{aligned}
$$

$\overline{\text { where } A_{c}}, B_{c}, K_{q}$ are controller gains, $B^{+}(\varphi)=$ $B^{T}(\varphi)$ $B^{T}(\varphi) B(\varphi)$ and $\delta K(t)$ represents the gain fluctuation which is described in the form $\delta K(t)=M_{K} \mathcal{F}(t) N_{K}$ where $M_{K}, N_{K}$ are known suitable dimensional matrices with $\mathcal{F}^{T}(t) \mathcal{F}(t) \leq I$ is Lebesgue measurable. Moreover, by the de-fuzzification process the following equations can be obtained,

$$
\begin{aligned}
\dot{x}_{c}(t) & =A_{c}(\varphi) x_{c}(t)+B_{c}(\varphi) y(t), \\
u(t) & =(K(\varphi)+\delta K(t)) x_{c}(t)-B^{+}(\varphi) B_{a}(\varphi) f_{O}(t),
\end{aligned}
$$

where $A_{c}(\varphi)=\sum_{p=1}^{l} \varphi_{p}(g(t)) A_{c p}, \quad B_{c}(\varphi)=$ $\sum_{p=1}^{l} \varphi_{p}(g(t)) B_{c p}$ and $\bigsqcup_{(\varphi)}=\sum_{q=1}^{l} \varphi_{q}(g(t)) K_{q}$. By substituting (17) into (5), the closed-loop is obtained in the following form,

$$
\begin{aligned}
\dot{x}(t)= & A(\varphi) x(t)+A_{d}(\varphi) x(t-\tau(t))+B(\varphi)(K(\varphi) \\
& +\delta K(t)) x_{c}(t)+D(\varphi) \omega(t)-B_{a}(\varphi) e_{f}(t),
\end{aligned}
$$

$$
y(t)=C x(t) .
$$

By defining the augmented vector $\eta(t)$ as $\left[\begin{array}{llll}x^{T}(t) & e^{T}(t) & x_{c}^{T}(t) & e_{f}^{T}(t)\end{array}\right]^{T}$ and combining the equations (11) - (19) the augmented form of T-S fuzzy system can be written as,

$$
\dot{\eta}(t)=A \eta(t)+A_{d} \eta(t-\tau(t))+D \omega(t)+B_{a} \dot{f}(t),
$$

$\begin{aligned} A= & {\left[\begin{array}{cccc}A(\varphi) & 0 & A_{13} & -B_{a}(\varphi) \\ 0 & (A(\varphi)+L(\varphi) C) & 0 & B_{a}(\varphi) \\ B_{c}(\varphi) C & 0 & A_{c}(\varphi) & 0 \\ 0 & A_{42} & 0 & A_{44}\end{array}\right], } \\ A_{13} & =\end{aligned}$ $-P_{4}^{-1} F(\varphi) C[A(\varphi)+L(\varphi) C+1], A_{44}=-P_{4}^{-1}$ $F(\varphi) C B_{a}(\varphi)$,

$A_{d}=\left[\begin{array}{cccc}A_{d}(\varphi) & 0 & 0 & 0 \\ 0 & A_{a}(\varphi) & 0 & 0 \\ 0 & 0 & 0 & 0 \\ 0 & -P_{4}^{-1} F(\varphi) C A_{d}(\varphi) & 0 & 0\end{array}\right]$,
$D=\left[\begin{array}{c}D(\varphi) \\ -D(\varphi) \\ 0 \\ P_{4}^{-1} F(\varphi) C D(\varphi)\end{array}\right]$ and $B_{a}=\left[\begin{array}{c}0 \\ 0 \\ 0 \\ -1\end{array}\right]$. In order to derive the required theoretical result, consider the following assumptions, definition and lemmas: 
Assumption 1. $\dot{f}(t)$ is norm bounded. i.e. $\|\dot{f}(t)\| \leq$ $f_{\max }$ where $0 \leq f_{\max }<\infty$.

Assumption 2. $\operatorname{Rank}\left(C B_{a_{p}}\right)=r$, where $r$ is the rank of $B_{a_{p}}$.

Assumption 3. Invariant zeros of $\left(A_{p}, B_{a_{p}}, C\right)$ lie in open left plane.

2.1. Lemma [12] Let the Assumption 2 and Assumption 3 hold then there exist a positive definite matrix $P_{2}$ such that the following condition holds, $F(\varphi) C=B_{a}^{T}(\varphi) P_{2}$.

2.2. Lemma [21] Let matrices $X, Y$ and $Z(t)$ be real matrices with appropriate dimensions and $Z(t)$ satisfying $Z^{T}(t) Z(t) \leq I$ is lebeque measurable, then for any scalar $\epsilon>0$, the following inequality holds, $X Z(t) Y+Y^{T} Z(t) X^{T} \leq \epsilon X X^{T}+\epsilon^{-1} Y^{T} Y$.

2.3. Definition [23] For given positive scalar $\gamma$ and $\theta \in[0,1]$, the constructed $T-S$ fuzzy system (19) achieves asymptotic stability with the performance mixed $H_{\infty}$ and passivity iff $\gamma \theta^{-1} y^{T}(t) y(t)-2(1-\theta) y^{T}(t) \omega(t)-\gamma \omega^{T}(t) \omega(t) \leq 0$ where, $\theta= \begin{cases}1 \text { if } H_{\infty} \\ 0 \text { if Passivity. }\end{cases}$

\section{Main results}

This section deals with the stability analysis of the prescribed system by emplpying L-K functional and adaptive algorithm. In order to do this, it is sufficient to prove that the system (5) is asymptotically stable with the prescribed performance index. To be precise, the following theorem presents the sufficient conditions guaranteeing the asymptotic stability of the system (5) without considering the gain fluctuations. From these conditions, the dynamic-output feedpack controller is obtained in Theorem (2) by considering gaip fluctuations. 3.1. Theorem Let the Assumptions 1-3 hold. For given positive scalars $\gamma$ and $\theta$, the system (5) is robustly asymptotically stable with mixed $H_{\infty}$ and passivity performance if there exist positive definite matrices $P_{1}, P_{2}$, $P_{3}, P_{4}, Q_{11}, Q_{11}, Q_{12}, Q_{13}, Q_{14}, Q_{21}, Q_{22}, Q_{23}, Q_{24}$, $Q_{31}, Q_{32}, Q_{33}, Q_{34}, M_{1}$ such that the following $L M I$ together with (22) hold:

$$
\begin{gathered}
{[\Xi]_{18 \times 18}=\left[\Xi_{i j}\right] \leq 0,} \\
F(\varphi) C=B_{a}^{T}(\varphi) P_{2},
\end{gathered}
$$

where $\Xi_{i, j}=\Xi_{j, i}, \forall i, j \in\{1,2, \ldots, 18\}, \Xi_{1,1}=$ $A^{T}(\varphi) P_{1}+P_{1} A(\varphi)+Q_{11}+Q_{21}+Q_{31}, \quad \Xi_{1,3}=$ $C^{T} B_{c}^{T}(\varphi) P_{3}+P_{1} B(\varphi) K(\varphi), \quad \Xi_{1,4}=-P_{1} B_{a}(\varphi)$, $\Xi_{1,5}=P_{1} D(\varphi)-(1-\theta) C^{T}, \Xi_{1,6}=P_{1} A_{d}(\varphi), \Xi_{1,18}=$ $\theta C^{T}, \quad \Xi_{2,2}=A^{T}(\varphi) P_{2}+P_{2} A(\varphi)+C^{T} L^{T}(\varphi) P_{2}+$ $P_{2} L(\varphi) C+Q_{12}+Q_{22}+Q_{32}, \Xi_{2,4}=-A(\varphi) P_{2} B_{a}(\varphi)-$ $C^{T} L^{T}(\varphi) P_{2} B_{a}(\varphi), \Xi_{2,5}=-P_{2} D(\varphi), \Xi_{2,6}=P_{2} A_{d}(\varphi)$, $\Xi_{3,3}=A_{c}^{T}(\varphi) P_{3}+P_{3} A_{c}(\varphi)+Q_{13}+Q_{23}+Q_{33}+M_{1}$, $\Xi_{4,4}=-2 B_{a}^{T}(\varphi) P_{2} B_{a}(\varphi)+Q_{14}+Q_{24}+Q_{34}+M$, $\Xi_{4,5}=B_{a}^{T}(\varphi) P_{2} D(\varphi), \quad \Xi_{4,6}=-B_{a}^{T}(\varphi) P_{2} A_{d}(\varphi)$, $\Xi_{5,5}=-\gamma I, \Xi_{6,6}=-(1-\mu) Q_{11}, \Xi_{7,7}=-(1-\mu) Q_{12}$, $\Xi_{8,8}=-(1-\mu) Q_{13}, \Xi_{9,9}=-(1-\mu) Q_{14}, \Xi_{10,10}=$ $-Q_{21}, \Xi_{11,11}=-Q_{22}, \Xi_{12,12}=-Q_{23}, \Xi_{13,13}=-Q_{24}$, $\Xi_{14,14}=-Q_{31}, \Xi_{15,15}=-Q_{32}, \Xi_{16,16}=-Q_{33}$, $\Xi_{17,17}=-Q_{34}, \Xi_{18,18}=-\gamma \theta$ and remaining terms are zeros. Moreover the error state of the fault are uniformly bounded.

\section{Proof:}

In order to prove the stability of the considered system (5), consider the following time-varying L-K functional,

$$
V(t)=\sum_{i=1}^{n} V_{p}(t), \quad \text { where }
$$

$$
\begin{aligned}
& V_{1}(t)=\eta^{T}(t) P \eta(t), \\
& V_{2}(t)=\int_{t-\tau(t)}^{t} \eta^{T}(s) Q_{1} \eta(s) d s, \\
& V_{3}(t)=\int_{t-\tau_{1}}^{t} \eta^{T}(s) Q_{2} \eta(s) d s, \\
& V_{4}(t)=\int_{t-\tau_{2}}^{t} \eta^{T}(s) Q_{3} \eta(s) d s,
\end{aligned}
$$

$P \quad=\quad \operatorname{diag}\left\{P_{1}, P_{2}, P_{3}, P_{4}\right\}, \quad Q_{p}=$ $\operatorname{diag}\left\{Q_{i 1}, Q_{i 2}, Q_{i 3}, Q_{i 4}\right\} \forall i \in\{1,2,3\}$. By taking derivatives of $V_{p}(t)$ 's along the trajectories of augmented system (20) we get,

$$
\begin{aligned}
\dot{V}_{1}(t)= & \dot{\eta}^{T}(t) P \eta(t)+\eta^{T}(t) P \dot{\eta}(t), \\
\dot{V}_{2}(t)= & \eta^{T}(t) Q_{1} \eta(t)-(1-\mu) \eta^{T}(t-\tau(t)) Q_{1} \\
& \times \eta(t-\tau(t)),
\end{aligned}
$$




$$
\begin{aligned}
& \dot{V}_{3}(t)=\eta^{T}(t) Q_{2} \eta(t)-\eta^{T}\left(t-\tau_{1}\right) Q_{2} \eta\left(t-\tau_{1}\right), \\
& \dot{V}_{4}(t)=\eta^{T}(t) Q_{3} \eta(t)-\eta^{T}\left(t-\tau_{2}\right) Q_{3} \eta\left(t-\tau_{2}\right) .
\end{aligned}
$$

Substitute the augmented system (20) into (24), we obtain

$$
\begin{aligned}
& \dot{V}_{1}(t)=2 \eta^{T}(t) P\left[A \eta(t)+A_{d} \eta(t-\tau(t))+D \omega(t)+B_{a}\right. \\
& \times \dot{f}(t)] \\
& =2 x^{T}(t) P_{1} A(\varphi) x(t)+2 x^{T}(t) P_{1} B(\varphi)(K(\varphi)+\delta K(t)) \\
& \times x_{c}(t)-2 x^{T}(t) P_{1} B_{a}(\varphi) e_{f}(t)-2 x^{T}(t) P_{1} A_{d}(\varphi) \\
& \times x(t-\tau(t))+2 x^{T}(t) P_{1} D \omega(t)+2 e^{T}(t) P_{2}[A(\varphi) \\
& +L(\varphi) C] e(t)+2 e^{T}(t) P_{2} B_{a}(\varphi) e_{f}(t)+2 e^{T}(t) P_{2} \\
& \times A_{d}(\varphi) e(t-\tau(t))-2 e^{T}(t) P_{2} D(\varphi) \omega(t)+2 x_{c}^{T}(t) \\
& \times P_{3} B_{c}(\varphi) C x(t)+2 x_{c}^{T}(t) P_{3} A_{c}(\varphi) x_{c}(t)-2 e_{f}^{T}(t) \\
& \times F(\varphi) C[A(\varphi)+L(\varphi) C+1] e(t)-2 e_{f}^{T}(t) F(\varphi) C \\
& \times B_{a}(\varphi) e_{f}(t)-2 e_{f}^{T}(t) F(\varphi) C A_{d}(\varphi) e(t-\tau(t)) \\
& +2 e_{f}^{T}(t) \widetilde{(\varphi)} C D(\varphi) \omega(t)-e_{f}^{T}(t) P_{3} \dot{f}(t) \text {. }
\end{aligned}
$$

From equation (22) the above equation becomes,

$$
\begin{aligned}
\dot{V}_{1}(t)= & 2 x^{T}(t) P_{1} A(\varphi) x(t)+2 x^{T}(t) P_{1} B(\varphi)(K(\varphi)+\delta K(t)) \\
& \times x_{c}(t)-2 x^{T}(t) P_{1} B_{a}(\varphi) e_{f}(t)-2 x^{T}(t) P_{1} A_{d}(\varphi) \\
& \times x(t-\tau(t))+2 x^{T}(t) P_{1} D \omega(t)+2 e^{T}(t) P_{2}[A(\varphi) \\
& +L(\varphi) C] e(t)+2 e^{T}(t) P_{2} A_{d}(\varphi) e(t-\tau(t))-2 e^{T}(t) \\
& \times P_{2} D(\varphi) \omega(t)+2 x_{c}^{T}(t) P_{3} B_{c}(\varphi) C x(t)+2 x_{c}^{T}(t) P_{3} \\
& \times A_{c}(\varphi) x_{c}(t)-2 e_{f}^{T}(t) B_{a}^{T}(\varphi) P_{2}[A(\varphi)+L(\varphi) C] e(t) \\
& -2 e_{f}^{T}(t) B_{a}^{T}(\varphi) P_{2} B_{a}(\varphi) e_{f}(t)-2 e_{f}^{T}(t) B_{a}^{T}(\varphi) P_{2} \\
& \times A_{d}(\varphi) e(t-\tau(t))+2 e_{f}^{T}(t) B_{a}^{T}(\varphi) P_{2} D(\varphi) \omega(t) \\
& -e_{f}^{T}(t) P_{3} \dot{f}(t) .
\end{aligned}
$$

For any positive definite matrix $M$ and based on Assumption 1, we can rewrite the last term of the above equation as follows

$$
\begin{aligned}
-2 e_{f}^{T}(t) P_{4} \dot{f}(t) & \leq e_{f}^{T}(t) M e_{f}(t)+\dot{f}^{T}(t) P_{4} M_{1}^{-1} P_{4} \dot{f}(t) \\
& \leq e_{f}^{T}(t) M e_{f}(t)+f_{\max }^{2} \lambda_{M},
\end{aligned}
$$

where $\lambda_{M}$ denotes ntaxintum eigen value of $P_{4} M_{1}^{-1} P_{4}$. From the equations (23)-(30) we have,

$$
\begin{aligned}
& \dot{V}(t)+\gamma \theta^{-1} y^{T}(t) y(t)-2(1-\theta) y^{T}(t) \omega(t)- \\
& \gamma \omega^{T}(t) \omega(t) \leq \Theta^{T}(t)[\Phi]_{17 \times 17} \Theta(t)+f_{\text {max }}^{2} \lambda_{M} \\
& \leq \Theta^{T}(t)[\Phi]_{17 \times 17} \Theta(t)+\Lambda,
\end{aligned}
$$

where $\Theta(t)=\left[\begin{array}{llll}\eta^{T}(t) & \omega^{T}(t) & \eta^{T}(t-\tau(t)) & \eta^{T}\left(t-\tau_{1}\right)\end{array}\right.$ $\left.\eta^{T}\left(t-\tau_{2}\right)\right]^{T}, \Phi_{i, j}=\Phi_{j, i}, \forall i, j \in\{1,2, \ldots, 17\}, \Phi_{1,1}=$
$A^{T}(\varphi) P_{1}+P_{1} A(\varphi)+Q_{11}+Q_{21}+Q_{31}+\gamma \theta^{-1} C^{T} C$, $\Phi_{1,3}=C^{T} B_{c}^{T}(\varphi) P_{3}+P_{1} B(\varphi)[K(\varphi)+\delta K(t)], \Phi_{1,4}=$ $-P_{1} B_{a}(\varphi), \Phi_{1,5}=P_{1} D(\varphi)-(1-\theta) C^{T}, \Phi_{1,6}=$ $P_{1} A_{d}(\varphi), \Phi_{2,2}=A^{T}(\varphi) P_{2}+P_{2} A(\varphi)+C^{T} L^{T}(\varphi) P_{2}+$ $P_{2} L(\varphi) C+Q_{12}+Q_{22}+Q_{32}, \Phi_{2,4}=-A(\varphi) P_{2} B_{a}(\varphi)-$ $C^{T} L^{T}(\varphi) P_{2} B_{a}(\varphi), \Phi_{2,5}=-P_{2} D(\varphi), \Phi_{2,6}=P_{2} A_{d}(\varphi)$, $\Phi_{3,3}=A_{c}^{T}(\varphi) P_{3}+P_{3} A_{c}(\varphi)+Q_{13}+Q_{23}+Q_{33}, \Phi_{4,4}=$ $-2 B_{a}^{T}(\varphi) P_{2} B_{a}(\varphi)+Q_{14}+Q_{24}+Q_{34}+M, \Phi_{4,5}=$ $B_{a}^{T}(\varphi) P_{2} D(\varphi), \Phi_{4,6}=-B_{a}^{T}(\varphi) P_{2} A_{d}(\varphi), \Phi_{5,5}=-\gamma I$, $\Phi_{6,6}=-(1-\mu) Q_{11}, \Phi_{7,7}=-(1-\mu) Q_{12}, \Phi_{8,8}=-(1-$ н) $Q_{13}, \Phi_{9,9}=-(1-\mu) Q_{14}, \Phi_{10,10}=-Q_{21}, \Phi_{11,11}=$ $-Q_{22}, \Phi_{12,12}=-Q_{23}, \Phi_{13,13}=-Q_{24}, \Phi_{14,14}=-Q_{31}$, $\Phi_{15,15}=-Q_{32}, \Phi_{16,16}=-Q_{33}, \Phi_{17,17}=-Q_{34}$, $\Lambda=f_{\max }^{2} \lambda_{M} P_{4}$ and remaining terms are zeros. By applying schur complement for the $\operatorname{trm} C^{T} C$, we can obtain the LMI which is given in (21), then we have $[\Phi]_{17 \times 17} \leq 0$ i.e. the eigen values of $[\Phi]_{17 \times 17}$ are negative, let the maximum among them is $-\Omega$ then

$$
\begin{aligned}
& \dot{V}(t)+\gamma \theta^{-1} y^{T}(t) y(t)-2(1-\theta) y^{T}(t) \omega(t)-\gamma \omega^{T}(t) \omega(t) \\
& \leq-\Omega\|\Theta(t)\|^{2}+\Lambda .
\end{aligned}
$$

From the above equation, it is clear that that $\dot{V}(t)+$ $\gamma \theta^{-1} y^{T}(t) y(t)-2(1-\theta) y^{T}(t) \omega(t)-\gamma \omega^{T}(t) \omega(t) \leq 0$ if and only if $\Lambda \leq \Omega\|\Theta(t)\|^{2}$ which implies that the error states of the fault are uniformly bounded. Under the zero initial conditions, we have $V(0)=0$ and $V(\infty)>0$ which implies that $\gamma \theta^{-1} y^{T}(t) y(t)-2(1-\theta) y^{T}(t) \omega(t)-$ $\gamma \omega^{T}(t) \omega(t) \leq 0$, then from the 2.3. Definition the above said inequality achieves mixed $H_{\infty}$ and passivity performance. By taking the noise term as zero we can show the asymptotic stability of the given system by using Lyapunov's stability theorem.

3.2. Remark cannot solve and find the unknown of the equation (22) by using LMI toolbox in Matlab. So we have to transform this equality into following LMI as the transformation of optimization problem

$$
\left(\begin{array}{ccc}
\rho I_{n} & B_{a}(\varphi)^{T} X_{2}-F(\varphi) C \\
* & \rho I_{n} & \square \square
\end{array}\right)>0 .
$$

3.3. Theorem Let the Assumptions 1-3 hold. For given positive scalars $\gamma, \rho, \theta$ the system (5) is robust asymptotically stable with mixed $H_{\infty}$ and passivity performance if there exist matrices $W_{1}, W_{2}, W_{3}, W_{4}$, positive definite matrices $X_{1}, X_{2}, X_{3}, P_{4}, Q_{11}, \hat{Q}_{11}, Q_{12}, \hat{Q}_{13}, Q_{14}, \hat{Q}_{21}$, $Q_{22}, \hat{Q}_{23}, Q_{24}, \hat{Q}_{31}, Q_{32}, \hat{Q}_{33}, Q_{34}, M$ and positive 
scalar $\epsilon$ such that the following LMIs are feasible,

$$
\begin{gathered}
{\left[\phi_{i, j}^{p p}\right]<0, p=q} \\
{\left[\phi_{i . j}^{p q}\right]+\left[\phi_{i, j}^{q p}\right]<0, p<q} \\
\left(\begin{array}{cc}
\rho I_{n} & B_{a_{p}}^{T} X_{2}-F(\varphi) C \\
* & \rho I_{n}
\end{array}\right)>0,
\end{gathered}
$$

where $i, j \in\{1,2, \ldots, 20\}, \phi_{i, j}^{p q}=\phi_{j, i}^{p q}, \forall p, q \in$ $\{1,2, \ldots, l\}, \phi_{1,1}^{p q}=X_{1} A_{p}^{T}+A_{p} X_{1}+\hat{Q}_{11}+\hat{Q}_{21}+\hat{Q}_{31}$, $\phi_{1,3}^{p q}=C^{T} W_{4 p}^{T}+B_{p} W_{1 q}, \phi_{1,4}^{p q}=-B_{a_{p}}, \phi_{1,5}^{p q}=D_{p}$, $\phi_{1,6}^{p q}=A_{d_{p}}, \phi_{1,18}^{p q}=X_{1} C^{T}, \phi_{1,19}^{p q}=\epsilon B_{p} M_{K}, \phi_{2,2}^{p q}=$ $A_{p}^{T} X_{2}+X_{2} A_{p}+C^{T} W_{2 p}^{T}+W_{2 p} C+Q_{12}+Q_{22}+Q_{32}$, $\phi_{2,4}^{p q}=-A_{p} X_{2} B_{a_{p}}-C^{T} W_{2 p}^{T} B_{a_{p}}, \phi_{2,5}^{p q}=-X_{2} D_{p}$, $\phi_{2,6}^{p q}=X_{2} A_{d_{p}}, \phi_{3,3}^{p q}=W_{3 p}^{T}+W_{3 p}+\hat{Q}_{13}+\hat{Q}_{23}+\hat{Q}_{33}$, $\phi_{3,20}^{p q}=X_{3} N_{K}^{T}, \phi_{4,4}^{p q}=-2 B_{a_{p}}^{T} X_{2} B_{a_{p}}+Q_{14}+Q_{24}+$ $Q_{34}+M, \phi_{4,5}^{p q}=B_{a_{p}}^{T} X_{2} D_{p}, \phi_{4,6}^{p q}=-B_{a_{p}}^{T} X_{2} A_{d_{p}}$, $\phi_{5,5}^{p q}=-\gamma I, \phi_{6,6}^{p q}=-(1-\mu) \hat{Q}_{11}, \phi_{7,7}^{p q}=-(1-\mu) Q_{12}$, $\phi_{8,8}^{p q}=-(1-\mu) \hat{Q}_{13}, \phi_{9,9}^{p q}=-(1-\mu) Q_{14}, \phi_{10,10}^{p q}=$ $-\hat{Q}_{21}, \quad \phi_{11,11}^{p q}=-Q_{22}, \quad \phi_{12,12}^{p q}=-\hat{Q}_{23}, \phi_{13,13}^{p q}=$ $-Q_{24}, \quad \phi_{14,14}^{p q}=-\hat{Q}_{31}, \phi_{15,15}^{p q}=-Q_{32}, \phi_{16,16}^{p q}=$ $-\hat{Q}_{33}, \phi_{17,17}^{p q}=-Q_{34}, \phi_{18,18}^{p q}=-I, \phi_{19,19}^{p q}=-\epsilon I$, $\phi_{20,20}^{p q}=-\epsilon I$ and remaining terms are zero. Further $K_{q}=W_{1 q} X_{3}^{-1}, L_{p}=X_{2}^{-1} W_{2 p}, A_{c i}=W_{3 p} X_{3}^{-1}$ and $B_{c_{p}}=W_{4 p} \mathcal{U S} X_{11}^{-1} \mathcal{S}^{-1} \mathcal{U}^{-1}$. Furthermore, the error states of the fault are uniformly bounded.

\section{Proof:}

Inspect and replace the following forms into (21): $A(\varphi)=\sum_{p=1}^{l} \varphi_{p}(g(t)) A_{p}, A_{d}(\varphi)=\sum_{p=1}^{l} \varphi_{p}(g(t)) A_{d p}$, $B(\varphi)=\sum_{p=1}^{l} \varphi_{p}(g(t)) B_{p}, B_{a}(\varphi)=\sum_{p=1}^{l} \varphi_{p}(g(t)) B_{a p}$, $D(\varphi)=\sum_{p=1}^{l} \varphi_{p}(g(t)) D_{p}, C=\sum_{p=1}^{l} \varphi_{p}(g(t)) C, A_{c}(\varphi)=$ $\sum_{p=1}^{l} \varphi_{p}(g(t)) A_{c p}, B_{c}(\varphi)=\sum_{p=1}^{l} \varphi_{p}(g(t)) B_{c p}, K(\varphi)=$ $\sum_{q=1}^{l} \varphi_{q}(g(t)) K_{q}, K_{q}=K_{q}+\delta K(t)$. Then (21) can be wrutten as,

$$
\left[\Xi_{i, j}\right]=\sum_{p=1}^{l} \sum_{q=1}^{l} \varphi_{p}(g(t)) \varphi_{q}(g(t)) \mathcal{Z}^{p q},
$$

where $\mathcal{Z}^{p q}=\left[\mathcal{Z}_{i, j}^{p q}\right], \mathcal{Z}_{i, j}^{p q}=\mathcal{Z}_{j, i}^{p q}, \forall i, j \in\{1,2, \ldots, 18\}$, $\mathcal{Z}_{1,1}^{p q}=A_{p}^{T} P_{1}+P_{1} A_{p}+Q_{11}+Q_{21}+Q_{31}, \mathcal{Z}_{1,3}^{p q}=$ $C^{T} B_{c_{p}}^{T} P_{3}+P_{1} B_{p}\left(K_{q}+\delta K(t)\right), \mathcal{Z}_{1,4}^{p q}=-P_{1} B_{a_{p}}, \mathcal{Z}_{1,5}^{p q}=$
$P_{1} D_{p}-(1-\theta) C^{T}, \mathcal{Z}_{1,6}^{p q}=P_{1} A_{d_{p}}, \mathcal{Z}_{1,18}^{p q}=\theta C^{T}, \mathcal{Z}_{2,2}^{p q}=$ $A_{p}^{T} P_{2}+P_{2} A_{p}+C^{T} L_{p}^{T} P_{2}+P_{2} L_{p} C+Q_{12}+Q_{22}+Q_{32}$, $\mathcal{Z}_{2,4}^{p q}=-A_{p} P_{2} B_{a_{p}}-C^{T} L_{p}^{T} P_{2} B_{a_{p}}, \mathcal{Z}_{2,5}^{p q}=-P_{2} D_{p}$, $\mathcal{Z}_{2,6}^{p q}=P_{2} A_{d_{p}}, \mathcal{Z}_{3,3}^{p q}=A_{c_{p}}^{T} P_{3}+P_{3} A_{c_{p}}+Q_{13}+Q_{23}+Q_{33}$, $\mathcal{Z}_{4,4}^{p q}=-2 B_{a_{p}}^{T} P_{2} B_{a_{p}}+Q_{14}+Q_{24}+Q_{34}+M$, $\mathcal{Z}_{4,5}^{p q}=B_{a_{p}}^{T} P_{2} D_{p}, \mathcal{Z}_{4,6}^{p q}=-B_{a_{p}}^{T} P_{2} A_{d_{p}}, \mathcal{Z}_{5,5}^{p q}=-\gamma I$, $\mathcal{Z}_{6,6}^{p q}=-(1-\mu) Q_{11}, \quad \mathcal{Z}_{7,7}^{p q}=-(1-\mu) Q_{12}$, $\mathcal{Z}_{8,8}^{p q}=-(1-\mu) Q_{13}, \quad \mathcal{Z}_{9,9}^{p q}=-(1-\mu) Q_{14}$, $\mathcal{Z}_{10,10}^{p q}=-Q_{21}, \mathcal{Z}_{11,11}^{p q}=-Q_{22}, \mathcal{Z}_{12,12}^{p q}=-Q_{23}$, $\mathcal{Z}_{13,13}^{p q}=-Q_{24}, \mathcal{Z}_{14,14}^{p q}=-Q_{31}, \mathcal{Z}_{15,15}^{p q}=-Q_{32}$, $\mathcal{Z}_{16,16}^{p q}=-Q_{33}, \mathcal{Z}_{17,17}^{p q}=-Q_{34}, \mathcal{Z}_{18,18}^{p q}=-\gamma \theta$ and the remaining terms are zeros. Define $\delta K(t)=M_{K} \mathcal{F}(t) N_{K}, X_{1}=P_{1}^{-1}, X_{2}=P_{2}, X_{3}=P_{3}^{-1}$, $\hat{Q}_{11}=X_{1} Q_{11} X_{1}, \hat{Q}_{21}=X_{1} Q_{21} X_{1}, \hat{Q}_{31}=X_{1} Q_{31} X_{1}$, $\hat{Q}_{13}=X_{3} Q_{13} X_{3}, \hat{Q}_{23}=X_{3} Q_{23} X_{3}, \hat{Q}_{33}=X_{3} Q_{33} X_{3}$, $W_{1 q}=X_{1} K_{q}, W_{2 p}=X_{2} L_{p}, W_{3 p}=X_{3} A_{c_{p}}$ and according to the Lemma 2 in [4], for $X_{3}=\mathcal{V} X \mathcal{V}^{T}$ there exists $\bar{X}_{3}=\mathcal{U S} X_{11} \mathcal{S}^{-1} \mathcal{U}^{-1}$ such that $C X_{3}=\bar{X}_{3} C$ where $X=\operatorname{diag}\left\{X_{11}, X_{22}, \ldots, X_{n n}\right\}$, $\bar{X}_{3}{ }^{-1}=\mathcal{U S} X_{11}^{-1} \mathcal{S}^{-1} \mathcal{U}^{-1}$ and $W_{4 p}=\square X_{3} B_{c_{p}}$. Make the pre and post multiplication for (35) with $\mathcal{M}=\operatorname{diag}\left\{X_{1}, I, X_{3}, I, I, X_{1}, I, X_{3}, I, X_{1}, I, X_{3}, I, X_{1}, I\right.$, $\left.X_{3}, I, I\right\}$ and by applying the 2.2. Lemma for the terms involving $\delta K(t)$ then it is easy to obtain the LMI terms in (33). By the 3.2. Remark, we can rewrite the equality (22) as in (34). Then the proof is obvious from the first theorem.

\section{Simulation Verifications}

In this section, a numerical example is presented to show the effectiveness of the proposed nonfragile fault-tolerance control.

Example : Let us consider the T-S fuzzy system (2) with two fuzzy rules and the system parameters as follows:

$$
\begin{aligned}
& A_{1} \quad=\quad\left[\begin{array}{ccc}
-10 & 10 & -1 \\
-0.8 & -1 & 0.1 \\
0.2 & 0 & -8 / 3
\end{array}\right], \quad A_{2}= \\
& \begin{array}{l}
{\left[\begin{array}{ccc}
-7 & 10 & -1 \\
-0.8 & -1 & 0.1 \\
0.1 & 0 & -8 / 3
\end{array}\right], B_{1}=\left[\begin{array}{c}
0.66 \\
0.9 \\
1
\end{array}\right], B_{2}=\left[\begin{array}{c}
0.69 \\
0.9 \\
1
\end{array}\right],} \\
A_{d_{1}}=0.01 A_{1}, A_{d_{2}}=0.01 A_{2}, C=\left[\begin{array}{lll}
0 & 1 & 0
\end{array}\right],
\end{array} \\
& D_{1}=\left[\begin{array}{c}
0 \\
0.1 \\
0.1
\end{array}\right], D_{2}=\left[\begin{array}{c}
0 \\
0.1 \\
0
\end{array}\right], M_{K}=\left[\begin{array}{lll}
0.1 & 0.01 & 0.2
\end{array}\right] \text {, }
\end{aligned}
$$


$N_{K}=0.05 I_{3}$.

The corresponding membership functions are selected as $\varphi_{1}(g(t))=\frac{1}{1+e^{x_{1}-0.5}}$ and $\varphi_{2}(g(t))=1-\varphi_{2}(g(t))$. The other vectors participated in the numerical simulation are taken as, $\tau_{1}=0, \tau_{2}=0.4, \mu=0.1, \rho=0.18$, $\gamma=0.09, \theta=0.8, \mathcal{F}(t)=\sin t I_{3}, \tau(t)=0.3+0.1 \sin t$, $\omega=0.5 \sin 7 t$ and $P_{4}=0.015$.

For the purpose of stabilization of the perturbed T$S$ fuzzy system (2), we design the time varying faulttolerant nonfragile controller as constructed in 17 with the above given parameters. Then, by solving the LMIs obtained in 3.3. Theorem, the following controller and observer gain matrices are obtained as

$$
\begin{aligned}
& K_{1}=\left[\begin{array}{lll}
0.0242 & -0.0406 & 0.7476
\end{array}\right], L_{1}=\left[\begin{array}{c}
-11.5193 \\
-9.3788 \\
-11.7501
\end{array}\right], \\
& A_{c_{1}}=\left[\begin{array}{ccc}
-1.9751 & -0.0973 & -0.0459 \\
0.0962 & -1.9753 & 0.1469 \\
0.0539 & -0.1491 & -2.0318
\end{array}\right], B_{c_{1}}=\left[\begin{array}{c}
-0.022( \\
0.0369 \\
-0.680 \leftarrow
\end{array}\right. \\
& K_{2}=\left[\begin{array}{lll}
-0.2780 & 0.2834-2.8035
\end{array}\right], L_{2}=\left[\begin{array}{c}
-7.2572 \\
-8.8008 \\
-8.6846
\end{array}\right], \\
& A_{c_{2}}=\left[\begin{array}{ccc}
-1.9980 & -0.1435 & -0.0611 \\
0.1888 & -1.9988 & 0.8379 \\
-0.3882 & -0.3808 & -4.2251
\end{array}\right], B_{c_{2}}=\left[\begin{array}{c}
0.2482 \\
-0.2530 \\
2.5029
\end{array}\right.
\end{aligned}
$$

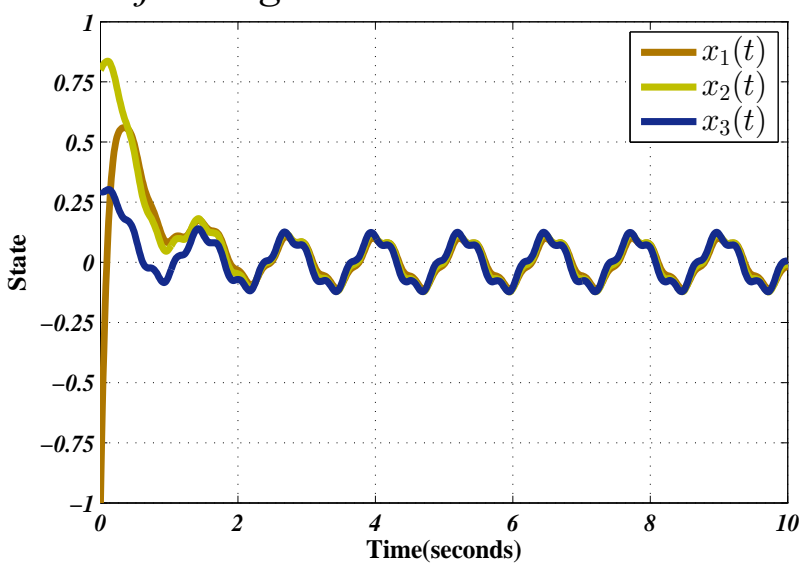

Fig. 2: State response for open-loop system

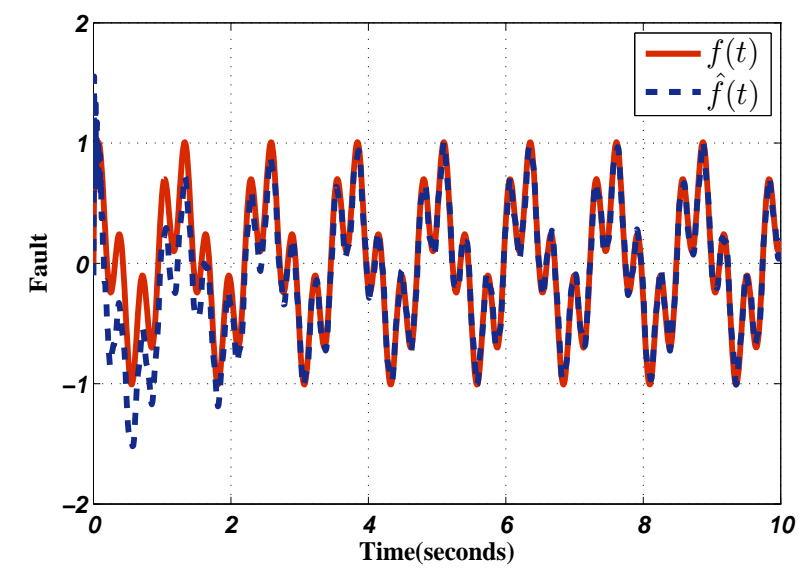

Fig. 3: Estimation of estimated fault with actual fault

$F_{1}=F_{2}=[2.9400]$.

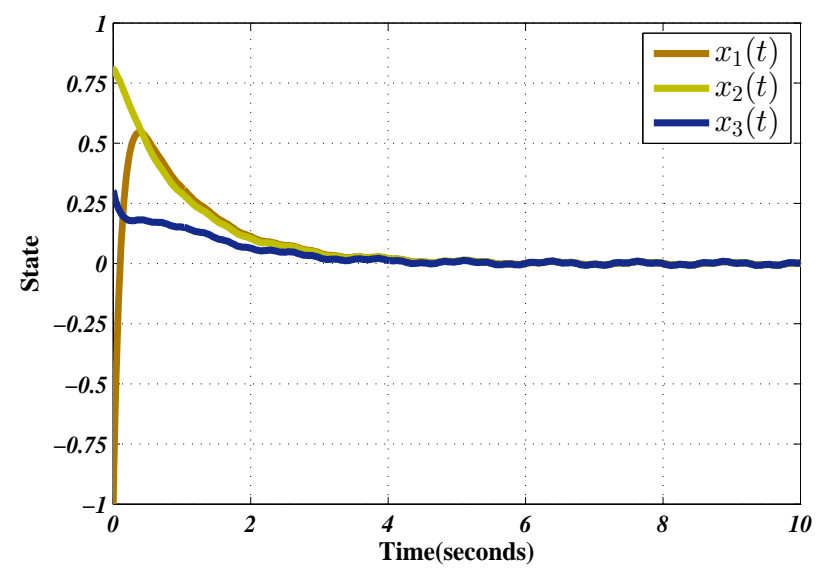

Fig. 1: State response for closed-loop system

For the fault $f(t)=1.5(0.4 \cos (5 t)+0.3 \sin (20 t))$ with the initial condition $\left[\begin{array}{lll}x_{1}(0) & x_{2}(0) & x_{3}(0)\end{array}\right]^{T}=$ $\left[\begin{array}{lll}-1 & 0.8 & 0.3\end{array}\right]^{T}$ and the obtained gain values, the state response of the closed-loop and open-loop system are displayed in the Fig. 1 and Fig. 2 respectively. In particular, from Fig. 1 it is obvious that the system attains stability with in 2-5 seconds even in the presence of timevarying actuator fault. It is noticed from the Fig. 3 the estimated fault tracks the original fault with maximum estimation error 0.8650 , which shows the effectiveness of prescribed fault-tolerance controller. The membership function is illustrated in Fig. 4 The output response with and without fault is displayed in Fig. 5.

Suppose, the fault is taken as $f(t)=\sin 10(t+1)-$ $3 e^{-10 *(t+2)^{2}+1} 1$, then from the Fig. 6 the estimation of fault with its estimated fault also have more accuracy. By observing the simulation studies, it is obvious that 


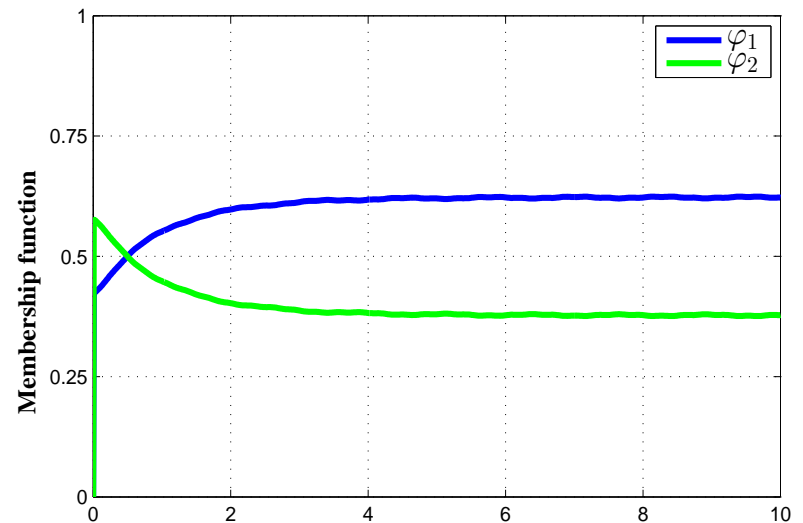

Fig. 4: Membership function

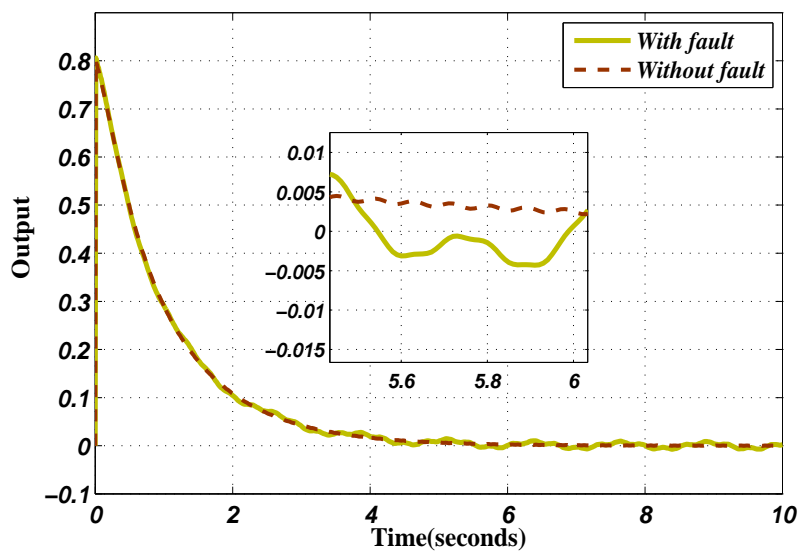

Fig. 5: Output response for with fault and without fault

the dynamic-output-feedback controller can promptly reclaim performance and stability of the considered closedloop even in the existence of disturbance and timevarying fault with precious estimation of the actuator faults.

\section{Conclusion}

In this paper, the problem of resilient reliable dynamicoutput-feedback $H_{\infty}$ control for the class of T-S fuzzy model with time varying fault and delay is investigated. Especially, the time-varying fault is dealt by using adaptive fault algorithm. In conjunction of L-K functional technique, S-procedure and Schur's lemma, a set of necessary and sufficient conditions has been obtained for asymptomatic stability of T-S fuzzy delayed system in the presence of time-varying actuator fault. Finally a numerical example is given to demonstrate the

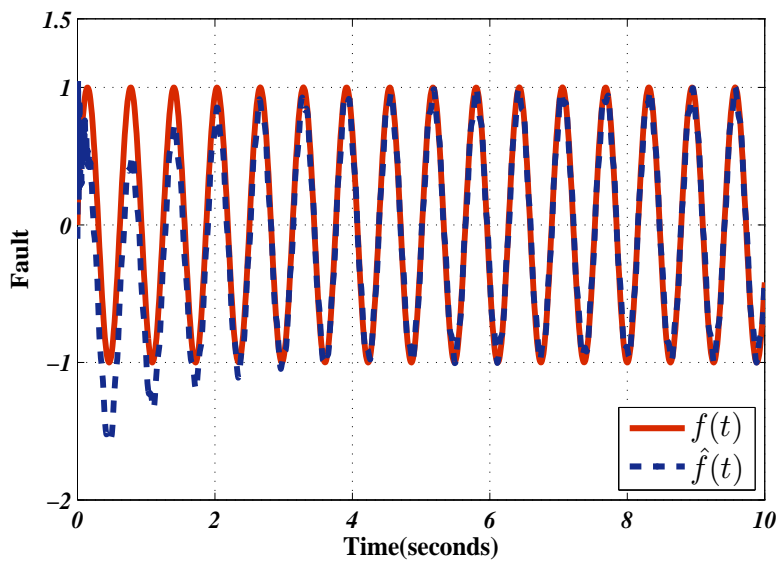

Fig. 6: Estimation of estimated fault with actual fault

effectiveness of the proposed controller.

\section{REFERENCES}

[1] Y. Liu, B.Z. Guo, J.H. Park and S. Lee. "Event-Based Reliable Dissipative Filtering for T-S Fuzzy Systems With Asynchronous Constraints", IEEE Transactions on Fuzzy Systems, volume 26, no. 4, pages 2089-2098, 2018.

[2] Y. Wang, H. Shen and H.R. Karimi, "Dissipativity-Based Fuzzy Integral Sliding Mode Control of Continuous-Time T-S Fuzzy Systems". IEEE Transactions on Fuzzy Systems, volume 26, no. 3, pages 1164 - 1176, 2018.

[3] X. Su, P. Shi, L. Wu, M.V. Basin. "Reliable Filtering With Strict Dissipativity for T-S Fuzzy Time-Delay Systems". IEEE Transactions on Cybernetics, volume 44, no. 12, pages 2470 2483, 2014.

[4] C. Peng, S. Ma, and X. Xie. "Observer-Based Non-PDC Control for Networked T-S Fuzzy Systems with an Event-Triggered Communication". IEEE Transactions on Cybernetics, volume 47, no. 8, pages 2279 - 2287, 2017.

[5] D. Zhang, Q.L. Han and X. Jia. "Network-Based Output Tracking Control for a Class of T-S Fuzzy Systems That Can Not Be Stabilized by Nondelayed Output Feedback Controllers". IEEE Transactions on Cybernetics, volume 45, no. 8, pages 15111523, Aug. 2015.

[6] H. Li, C. Wu, S. Yin and H.K. Lam. "Observer-Based Fuzzy Control for Nonlinear Networked Systems Under Unmeasurable Premise Variables", IEEE Transactions on Fuzzy systems, Vol. 24, no. 5, pages 1233-1245, 2016.

[7] X. Zhao, Y. Yin, L. Zhang and H. Yang. "Control of Switched Nonlinear Systems via T-S Fuzzy Modeling ”. IEEE Transactions on Fuzzy systems, volume 24, no. 1, pages 235-241, 2016.

[8] W. Xiong, W. Yu, J. Lu and X. Yu. "Fuzzy Modelling and Consensus of Nonlinear Multiagent Systems With Variable Structure". IEEE Transactions on Circuits and Systems, volume 61, no. 4, pages 1183-1191, 2014. 
[9] O. M. Kwon, M. J. Park, J. H.Park and S.M. Lee. "Stability and stabilization of TS fuzzy systems with time-varying delays via augmented Lyapunov-Krasovskii functionals". Information Sciences, volume 372, no. 1, pages 1-15, 2016.

[10] Z. Lian, Y. He, C.K. Zhang and M. Wu. "Stability analysis for T-S fuzzy systems with time-varying delay via free-matrixbased integral inequality". International Journal of Control, Automation and Systems, volume 14, no. 1, pages 21-28, Feb. 2016.

[11] L. Li, M. Chadli, S.X. Ding, J. Qiu and Y. Yang, "Diagnostic Observer Design for TS Fuzzy Systems: Application to RealTime-Weighted Fault-Detection Approach ”. IEEE Transactions on Fuzzy Systems, volume 26, no. 2, pages 805-816, April. 2018.

[12] F. You, H. Li, F. Wang and S. Guan. "Robust Fast Adaptive Fault Estimation for Systems with Time-Varying Interval Delay", Journal of the Franklin Institute, volume 352, no. 12 pages 5486-5513, 2015.

[13] T. Youssef, M. Chandli, H.R. Karimi and R. Wang. "Actuator and sensor faults estimation based on proportional integral observer for TS fuzzy model". Journal of the Franklin Institute, volume 354, no. 6, pages 2524-2542, 2017.

[14] S.H. Huang and G.H. Yang. "Fault Tolerant Controller Design for TS Fuzzy Systems With Time-Varying Delay and Actuator Faults: A K-Step Fault-Estimation Approach ”. IEEE Transactions on Fuzzy systems, volume 22, no. 6, pages 1526-1540, 2014.

[15] H. Yang, Y. Xia and B. Liu. "Fault Detection for TS Fuzzy Discrete Systems in Finite-Frequency Domain”. IEEE Transactions on Systems, Man, and Cybernetics, Part B (Cybernetics), volume 41, no. 4, pages 911-920, 2011.

[16] X. J. Li and G.H. Yang. "Fault Detection in Finite Frequency Domain for Takagi-Sugeno Fuzzy Systems With Sensor Faults ", IEEE Transactions on Cybernetics, volume 44, no. 8, pages 1446-1458, 2014.

[17] D. Ichalal, B. Marx, J. Ragot and D. Maquin. "Fault detection, isolation and estimation for TakagiSugeno nonlinear systems ". Journal of the Franklin Institute, volume 351, no. 7, pages 3651-3676, July 2014.

[18] X. Hu, L. Wu, C. Hu, Z. Wang and H. Gao. "Dynamic output feedback control of a flexible air-breathing hypersonic vehicle via TS fuzzy approach ". International Journal of Systems Science, volume 45, no. 8, pages 1740-1756, Aug. 2014.

[19] W. Zheng, Z.M. Zhang, H.B. Wang, H.R. wang and P.H. Yin. "Stability Analysis and Dynamic Output Feedback Control for Nonlinear T-S Fuzzy System with Multiple Subsystems and Normalized Membership Functions". International Journal of Control, Automation and Systems, volume16, no. 6, pages 2801-2813, 2018.

[20] H. D. Choi, C. K. Ahn, P. Shi, L. Wu, M. T. Lim. "Dynamic output-feedback dissipative control for TS fuzzy systems with time-varying input delay and output constraints". IEEE Transactions on Fuzzy Systems, volume 25, no. 3, pages 511 - 526, 2017.

[21] X.H. Chang, J. Xiong and J.H. Park. "Fuzzy robust dynam- ic output feedback control of nonlinear systems with linear fractional parametric uncertainties ". Applied Mathematics and Computation, volume 291, no. 1, pages 213-225, 2016.

[22] D.H. Lee, J.B. Park, Y.H. Joo and S.K. Kim. "Local $H_{\infty}$ controller design for continuous-time T-S fuzzy systems". International Journal of Control, Automation and Systems, volume 13, no. 6, pages 1499-1507, 2015.

[23] P. Shi, M. Chadli, Z. Xi and R. K. Agarwal. "Mixed H-Infinity and Passive Filtering for Discrete Fuzzy Neural Networks with Stochastic Jumps and Time Delays ". IEEE Transactions on neural networks and learning systems, volume 27 , no. 4, pages 903-909, 2016.

[24] Q. Gao, G. Feng, Z. Xi , Y. Wang and J. Qiu. "A New Design of Robust $H_{\infty}$ Sliding Mode Control for Uncertain Stochastic T-S Fuzzy Time-Delay Systems". IEEE Transactions on Cybernetics, volume 44, no. 9, pages 1556-1566, 2014. 